\title{
Deposition of Thermoelectric Strontium Hexaboride Thin Films by a Low Pressure CVD Method
}

\author{
Tommi Tynell, Takashi Aizawa, Isao Ohkubo, Katsumitsu Nakamura and Takao Mori* \\ International Center for Materials Nanoarchitectonics (MANA), National Institute for Materials \\ Science (NIMS), 1-1 Namiki, 305-0044 Tsukuba, Japan
}

\begin{abstract}
Thin films of $\mathrm{SrB}_{6}$ were deposited on sapphire substrates using a chemical vapor deposition method, with elemental strontium and decaborane, $\mathrm{B}_{10} \mathrm{H}_{14}$, used as the strontium and boron sources, respectively. The formation of highly crystalline, phase-pure $\mathrm{SrB}_{6}$ films was confirmed with X-ray diffraction and reflection high energy diffraction (RHEED) analysis, and the films' thermoelectric transport properties were measured. A relatively high deposition temperature of 850 $950{ }^{\circ} \mathrm{C}$ was found to be optimal for obtaining well-crystallized films at an extremely high deposition rate. The thermoelectric transport properties of the $\mathrm{SrB}_{6}$ thin films were observed to be comparable to those reported for bulk materials, but an unexpectedly high electrical resistivity led to a reduced power factor value for the thin films.
\end{abstract}

KEYWORDS: A3. Chemical vapor deposition processes, B1. Strontium compounds, B1. Borides, B2. Thermoelectric

${ }^{*}$ Corresponding Author:

Takao Mori

International Center for Materials Nanoarchitectonics

National Institute for Materials Science

1-1 Namiki, Tsukuba, Ibaraki, 305-0044 Japan

e-mail: MORI.Takao@nims.go.jp 


\section{Introduction}

Boron-rich metallic borides are known for often having very complex crystal structures, but hexaborides, $M \mathrm{~B}_{6}$, form an exception with their relatively simple cubic crystal structure. Despite this structural simplicity, metallic hexaborides have been found to exhibit a number of interesting properties, such as the ferromagnetism observed in $\mathrm{EuB}_{6}[1]$ and, more controversially, La-doped $\mathrm{CaB}_{6}[2,3]$, or the low work function and vacuum stability which makes hexaborides such as $\mathrm{LaB}_{6}$ or $\mathrm{CeB}_{6}$ suitable for use as hot cathode materials in electron field emission applications [4,5]. Alkaline earth hexaborides have also been identified as n-type thermoelectric materials, and a thermoelectric figure of merit as high as 0.35 has been reported for their bulk samples [6-10]. This performance along with the borides' general high capability to withstand high temperatures makes alkaline earth hexaborides among the promising materials [11] for possible usage in high temperature thermoelectric energy harvesting applications such as topping cycles of thermal power plants [12], where conventional state of the art materials are unusable. However, improving the thermoelectric performance of the materials remains a priority if they are to be used in applications on a large scale. Nanostructuring approaches such as the fabrication of layered superlattices offer a straightforward way to achieve improvements in thermoelectric performance even at room temperature [13-15], but a suitable thin film process is required to realize this goal.

Previous studies on hexaboride thin film depositions have largely concentrated on $\mathrm{LaB}_{6}$ and $\mathrm{CeB}_{6}$ [16,17], and $\mathrm{CaB}_{6}$ thin film processes have also been studied on several occasions [18,19], but $\mathrm{SrB}_{6}$ thin films have seen relatively little study. A previous study has looked at the thermoelectric performance of alkaline earth hexaboride thin films deposited via pulsed laser deposition [20], but otherwise the thermoelectric properties of hexaboride thin films have remained unexplored. Chemical vapor deposition (CVD) offers a robust process for depositing boron films through the use of various boron hydride precursors [21,22], and the method has been utilized before to deposit thin films of borides such as $\mathrm{LaB}_{6}$ [23], $\mathrm{NdB}_{6}$ [24] and $\mathrm{MgB}_{2}$ [25]. While CVD generally utilizes volatile compounds that react to form the desired thin film, the $\mathrm{MgB}_{2}$ study reported that an elemental $\mathrm{Mg}$ source can be used together with decaborane in a process to deposit boride films [25]. Here we

demonstrate that a similar method, based on elemental strontium and decaborane sources, can be used to deposit films of $\mathrm{SrB}_{6}$ and report the thermoelectric properties of the films. 


\section{Materials and Methods}

Thin films of $\mathrm{SrB} 6$ were deposited on single-crystal sapphire (0001) substrates (Shinkosha) using a custom-built CVD reactor. The reactor consisted of a molybdenum sample stage/heater and two heated precursor sources: one valve-controlled source containing decaborane $\left(\mathrm{B}_{10} \mathrm{H}_{14}\right)$ and one source consisting of an open $\mathrm{Al}_{2} \mathrm{O}_{3}$ crucible with a tungsten heating wire within the reaction chamber. Hence, the decaborane flow into the reaction chamber could be controlled with the valve, but the Sr flow onto the substrate was determined entirely by its heating temperature. The walls of the reaction chamber where unheated, and the system was kept at a base pressure of around $10^{-3} \mathrm{~Pa}$ using a turbomolecular pump. The films were deposited using substrate temperatures of $700-950{ }^{\circ} \mathrm{C}$, while the source temperatures for decaborane and $\mathrm{Sr}$ were kept at $110^{\circ} \mathrm{C}$ and $750-770{ }^{\circ} \mathrm{C}$, respectively. It should be noted that the reported temperature for the Sr source was measured from the bottom of the crucible, so the actual $\mathrm{Sr}$ temperature could be as much as $200^{\circ} \mathrm{C}$ lower. Thus, the vapor pressure of the heated $\mathrm{Sr}$ metal was expected to be in the 1-10 Pa range, which would result in quite a strong $\mathrm{Sr}$ flow onto the substrate. While precise precursor flows could not be measured, the pressure in the reaction chamber (measured using an ion gauge) was monitored and kept constant at 2-3 $\times 10^{-2} \mathrm{~Pa}$ by adjusting the decaborane precursor valves. The deposition time was 10 minutes for all the depositions, which already resulted in films around 2.5-3.5 $\mu \mathrm{m}$ thick, corresponding to a very fast deposition rate of $0.25-0.35 \mu \mathrm{m} / \mathrm{min}$.

For structural characterization, X-ray diffraction (XRD) measurements were performed using a Rigaku Rind-Ultima III X-ray diffractometer in 2 $\theta / \theta$ mode. For one of the samples, the surface crystal structure was also studied using a reflection high energy electron diffraction (RHEED) apparatus, performed in ultra-high vacuum conditions of around $10^{-8} \mathrm{~Pa}$. This sample was heated to $600{ }^{\circ} \mathrm{C}$ for two hours inside the vacuum chamber of the RHEED system in order to eliminate potential surface contamination during exposure to the atmosphere. The thicknesses of the $\mathrm{SrB}_{6}$ films were determined by using a Dektak 6M surface profiler system and confirmed with a JEOL JSM-6700F scanning electron microscope (SEM). A Rutherford backscattering spectrometry (RBS) setup was also used to evaluate the distribution of the elements within the films. Finally, the thermoelectric transport properties (Seebeck coefficient and electrical resistivity) were simultaneously measured with an ULVAC ZEM-2 apparatus from room temperature to $700{ }^{\circ} \mathrm{C}$ under a helium atmosphere. 


\section{Results and Discussion}

Black films with a uniform, opaque appearance were obtained across the investigated substrate temperature range of $700-950{ }^{\circ} \mathrm{C}$. The formation of the $\mathrm{SrB}_{6}$ structure was confirmed for all of the samples using XRD, with the XRD pattern of the $950{ }^{\circ} \mathrm{C}$ sample displayed in Figure 1. The indexed peaks in the figure correspond to those resulting from the cubic structure of $\mathrm{SrB}_{6}$, while the unindexed peaks are all from the sapphire substrate (including some impurities already present in the substrate). Thus, the films consisted of pure $\mathrm{SrB}_{6}$, with no impurity phases detected, indicating a successful fabrication of $\mathrm{SrB}_{6}$ thin films. There was also no shifting of diffraction peaks observed, which was expected because the films are so thick that any lattice strain inflicted upon the film by the substrate would be expected to be accommodated through defect formation mechanisms at a critical thickness far lower than the 2.5-3.5 $\mu \mathrm{m}$ deposited in this work. The lattice constant value of $4.194 \AA$ calculated from the XRD pattern also closely matches the reported value for bulk $\mathrm{SrB}_{6}$ of $4.198 \AA$ [26], a difference of approximately $0.1 \%$. The two main sapphire diffraction peaks are cut off from the pattern in Figure 1 to make the film diffraction pattern visible in the figure; the intensity of the (100) SrB6 peak was approximately $1-10 \%$ of the intensity of the sapphire (0006) peak, varying with the deposition temperature.

The most striking feature of the XRD pattern in Figure 1 is how dominant the (100) direction diffractions are compared with the other peaks. Although all the expected $\mathrm{SrB}_{6}$ diffractions are discernible, their peak intensities are far below those of the (100) peaks, indicating a strong preferred orientation towards the $<100>$ direction. The (110) peak especially is strongly suppressed; in a bulk powder diffraction pattern it would be expected to be the strongest peak, yet here it is barely $1 / 10$ of the (100) peak intensity. The XRD patterns of all the analyzed samples were very similar to the one shown in Figure 1, although the intensities of the diffraction peaks, particularly the (100) peak, varied with the deposition temperature.

The obtained XRD patterns were also used to observe changes in the crystallinity of the $\mathrm{SrB}_{6}$ films based on the intensity and width of the (100) diffraction peak. Since a change in diffraction intensity under identical measurement conditions can be thought to arise either from a change in the crystallinity or thickness of the analyzed thin film, the evolution of the $\mathrm{SrB}_{6}$ film crystallinity can be estimated by comparing the intensity of the (100) diffraction peak normalized in relation to the film thickness and sapphire (0006) peak intensity according to the formula $I_{\mathrm{Norm}(100)}=I_{\mathrm{SB}(100)} / I_{\mathrm{Sapph}(0006)} d$, where $d$ is the film thickness. Likewise, the width of the diffraction peak can be used to estimate the crystallinity of the film, with narrower peaks indicating larger grains and thus a higher crystalline 
quality. The normalized (100) peak intensities and measured peak widths at half maximum for films deposited between 700 and $950{ }^{\circ} \mathrm{C}$ are plotted in Figure 2.

The normalized (100) peak intensity can be seen to rise with increasing deposition temperature until $850{ }^{\circ} \mathrm{C}$, signifying an improving degree of crystallinity. After $850{ }^{\circ} \mathrm{C}$ the intensity starts to go down slightly, which could result from decreasing crystallinity. However, the differences between the higher temperature samples are rather small compared to the roughness of the used method of approximating changes in crystallinity, so the observed intensity decrease may not reflect an actual decrease in crystallinity. The (100) peak width displays a very similar trend, decreasing as the deposition temperature increases, thus indicating improving crystalline quality, before stabilizing at $850{ }^{\circ} \mathrm{C}$. Therefore, the data in Figure 2 is interpreted as demonstrating that the film crystallinity increases with the deposition temperature until $850{ }^{\circ} \mathrm{C}$, and well-crystallized films are obtained at deposition temperatures above that.

To confirm the XRD-based conclusions on film crystallinity, a RHEED pattern was recorded under ultra-high vacuum conditions from the surface of the sample deposited at $950{ }^{\circ} \mathrm{C}$. The obtained diffraction pattern is shown in Figure 3. Although the diffraction spots visible in Figure 3 are rather fuzzy, the pattern clearly matches that of a cubic lattice, confirming the XRD observations of a highly (100)-oriented $\mathrm{SrB}_{6}$ film. Furthermore, the appearance of clearly defined diffraction spots as seen in the RHEED pattern indicates that the film is well crystallized, although the fuzzy appearance of the spots is a sign that the film surface is not smooth. The pattern also changed very little upon changing the azimuth of the electron beam. This observation, along with the arc-like nature of the diffraction spots, indicates that the film is polycrystalline, consisting of grains with a preferred orientation in the $<100>$ direction.

The RHEED observations together with the highly suppressed diffraction peaks of the XRD pattern indicate that the film growth is columnar in nature, with a high orientation preference for the (100) direction. To confirm this, cross-sectional SEM images were taken from samples broken in half for this purpose, and typical images are shown in Figure 4. The images clearly show a polycrystalline film with a texture that indicates columnar growth perpendicular to the film surface. The brighter lines visible at the interfaces of the film are thought to result from edge effects rather than any compositional difference with the rest of the film. The images were also used to confirm the thickness values of the deposited films, which were found to match the values obtained with the surface profiler apparatus. 
Comparing the deposition rate of the $\mathrm{SrB}_{6}$ films, $0.25-0.35 \mu \mathrm{m} / \mathrm{min}$, to rates reported for a boron deposition process based on decaborane [22] reveals that the values obtained in this work are very similar to the boron process under the used process conditions. However, the boron films deposited through decaborane pyrolysis were amorphous, whereas a highly crystalline $\mathrm{SrB}_{6}$ film is obtained in the present study when elemental strontium is added into the process. This suggests that the reaction between strontium and boron in the described CVD process is extremely fast, resulting in rapid crystal growth, and that the pyrolysis of decaborane into boron is the limiting step in the film growth. Compared to other boride processes, the determined rate of $0.25-0.35 \mu \mathrm{m} / \mathrm{min}$ is far higher than rates reported in previous studies on the $\mathrm{CVD}$ of $\mathrm{LaB}_{6}$ [23], $\mathrm{NdB}_{6}$ [24] or $\mathrm{MgB}_{2}$ [25], which reported growth rates of $0.4-2 \mu \mathrm{m} / \mathrm{h}(0.007-0.03 \mu \mathrm{m} / \mathrm{h})$. In the case of the lanthanoid hexaboride processes, the higher deposition rate of $\mathrm{SrB}_{6}$ makes sense, since those processes were based on chloride sources for the lanthanoid element, so the lack of a need to break the bond between the metal and halogen atoms could be expected to lead to a faster growth rate in a process based on an elemental metal source $[23,24]$. The differences with the $\mathrm{MgB}_{2}$ report cannot be explained with different source materials, as an elemental source was used in that study as well. However, the pressures used in our setup were approximately two magnitudes higher than those utilized in the $\mathrm{MgB}_{2} \mathrm{CVD}$ study, which can explain the very different growth rate [25]. Therefore, the highly reactive source materials combined with the high source gas pressures are thought to be the reason behind the extremely high growth rates observed.

The characterization of the films' thermoelectric transport properties was focused on samples deposited between $850-950{ }^{\circ} \mathrm{C}$ due to the well-crystallized nature of the films. No differences in film properties were observed between samples deposited in this temperature range, and the obtained results for the thermoelectric transport properties measured between room temperature and $700{ }^{\circ} \mathrm{C}$ are presented in Figure 5. The values for both resistivity and Seebeck coefficient are comparable to those reported for bulk samples, although the resistivity value is about one magnitude higher than in the bulk [8]. The temperature behavior of the resistivity value is also different from that reported for bulk samples; while the trend of rising resistivity with temperature is common for both bulk and thin film samples, the slope of the graph suddenly increases in higher temperature for the film samples, as can be seen in Figure 5. Thus the difference between film and bulk properties is especially large at high temperatures. The Seebeck coefficient properties display the same trend in the thin film samples as reported for bulk, with the only difference being that the value of the Seebeck coefficient is slightly lower in the films. The transport property values obtained here are also similar to ones reported for SrB6 films deposited using a pulsed laser deposition technique [20], but again the resistivity value is 
approximately 4-5 times higher than previously reported. Curiously, an opposite trend was reported for the temperature behavior of resistivity in $\mathrm{SrB}_{6}$ films, namely that the resistivity was seen to decrease with increasing temperature [20]. It is unclear what caused that observation, but the currently seen trend of increasing resistivity is consistent with bulk behavior as well as the trend of the Seebeck coefficient.

The Seebeck coefficient and resistivity values were used to calculate the thermoelectric power factor of the $\mathrm{SrB}_{6}$ thin films, which is shown in the lower part of Figure 5. Due to the rapidly increasing resistivity of the films, the highest power factor value of $7.5 \times 10^{-5} \mathrm{~W} / \mathrm{mK}^{2}$ is reached at $400{ }^{\circ} \mathrm{C}$, which is more than a magnitude lower than the best bulk values reported [8]. The low temperature at which the films' performance peaks is rather unfortunate, since one of the advantages of using boride materials is their ability to withstand high temperatures, and is also different from the bulk sample behavior in literature, where the power factor was found to increase with temperature, simply because the Seebeck coefficient was rising faster than the resistivity, resulting in an overall improvement in performance [8].

Since the Seebeck coefficient is mostly unchanged from bulk values, the high resistivity values observed in the $\mathrm{SrB}_{6}$ films can be seen to result from lower electron mobility rather than a difference in carrier concentration. The cause of this decrease in carrier mobility is thought to be an increase in impurities or grain boundaries in the thin film samples, which was not unexpected because thin films generally have a far greater concentration of grain boundaries than equivalent bulk materials. This view is supported by the SEM image in Figure 4, which shows a film with a high density of thin, columnar grains. It can also explain the differences between the resistivity values observed here and in the previous study on $\mathrm{SrB} 6$ films, since the different deposition method and substrate materials can be expected to result in very different film grain structures. Another possibility for the cause of the high resistivity would be the existence of amorphous boron phases within the film or the uneven distribution of strontium. To shed light on this, an RBS measurement was performed on a sample deposited at $850{ }^{\circ} \mathrm{C}$. While the measurement was inconclusive regarding the elemental ratio of $\mathrm{Sr}$ and $\mathrm{B}$, it did show that Sr was very evenly distributed across the film. This observation, along with the columnar, polycrystalline grain structure seen in the SEM image, suggests that the main cause of high resistivity in the deposited $\mathrm{SrB}_{6}$ films is the high density of grain boundaries within the films.

In terms of thermoelectric performance, the temperature behavior of the power factor seen in Figure 7 does not necessarily mean that the thin films perform the best at $400{ }^{\circ} \mathrm{C}$, since the temperature behavior of the thermal conductivity of the material is expected to closely follow the same pattern to 
its electrical conductivity. In fact, the lattice thermal conductivity, which is not related to free electrons, would be expected to be affected by impurities and grain boundaries to the same or larger extent than conductivity based on electrons due to the generally larger mean free path of phonons. Thus, to get a full picture of the thin films' thermoelectric performance, their thermal conductivities should be determined, which will be the subject of a further study.

\section{Conclusions}

We have fabricated highly textured thin films of $\mathrm{SrB} 6$ on sapphire substrates from elemental strontium and decaborane sources using a simple CVD-based method. The films display extremely high growth rates of $0.25-0.35 \mu \mathrm{m} / \mathrm{min}$, which is thought to be the result of the use of highly reactive source materials and high source gas pressures. Although films can be deposited at temperatures as low as $700{ }^{\circ} \mathrm{C}$, a deposition temperature of $850{ }^{\circ} \mathrm{C}$ is necessary to obtain fully crystalline films. While the Seebeck coefficient values of the films are very similar to those reported for bulk samples, the resistivity was found to be surprisingly high, especially at high temperatures, resulting in a thermoelectric power factor approximately a magnitude lower than in the bulk, with the performance peaking already at $400{ }^{\circ} \mathrm{C}$. The reason behind the high resistivity is thought to be decreased electron

mobility due to a higher concentration of grain boundaries in the films resulting from highly oriented, columnar growth of small crystallites of $\mathrm{SrB} 6$.

\section{Acknowledgements}

The authors would like to acknowledge funding from the Japan Society for the Promotion of Science (JSPS) under the JSPS Postdoctoral Fellowship for Foreign Researchers program. TM, TA, IO are also supported by CREST, JST. The authors would also like to thank Ryosuke Maki for assistance with the SEM analysis. 


\section{References}

1. S. Sullow, I. Prasad, M.C. Aronson, J.L. Sarrao, Z. Fisk, D. Hristova, A.H. Lacerda, M.F. Hundley, A. Vigliante and D. Gibbs, Phys. Rev. B 57, 5860 (1998).

2. D. P. Young, D. Hall, M. E. Torelli, Z. Fisk, J. L. Sarrao, J. D. Thompson, H. R. Ott, S. B. Oseroff, R. G. Goodrich and R. Zysler, Nature 397, 412 (1999).

3. T. Mori and S. Otani, Solid State Commun. 123, 287 (2002).

4. C. Oshima, E. Bannai, T. Tanaka, and S. Kawai, J. Appl. Phys. 48, 3925 (1977).

5. J. Xu, G. Hou, T. Mori, H. Li, Y. Wang, Y. Chang, Y. Luo, B. Yu, Y. Ma, and T. Zhai, Adv. Funct. Mater. 23, 5038 (2013).

6. Yu. B. Paderno, Poroshk. Metallurgija 11, 70 (1969).

7. J. Etourneau, J. P. Mercurio, R. Naslain and P. Hagenmuller, J. Solid State Chem. 2, 332 (1970).

8. M. Takeda, M. Terui, N. Takahashi and N. Ueda, J. Solid State Chem. 179, 2823 (2006).

9. K. Yagasaki, S. Notsu, Y. Shimoji, T. Nakama, R. Kaji, T. Yokoo, J Akimitsu, M. Hedo and Y. Uwatoko, Physica B 329-333, 1259 (2003).

10. M. Gürsoy, M. Takeda and B. Albert, J. Solid State Chem. 221, 191 (2015).

11. T. Mori, "Higher Borides" in Handbook on the Physics and Chemistry of Rare Earths, Vol. 38, ed. K. A. Gschneidner, J.-C. G. Bünzli, and V. K. Pecharsky (Elsevier B. V., Amsterdam, 2008) pp. 105-173.

12. K. Yazawa and A. Shakouri, Scr. Mater. 111, 58 (2016).

13. L. D. Hicks and M. S. Dresselhaus, Phys. Rev. B 47(19), 12727 (1993).

14. C. J. Vineis, A. Shakouri, A. Majumdar and M. G. Kanatzidis, Adv. Mater. 22, 3970 (2010).

15. W. Liu, Z. Ren and G. Chen, in Thermoelectric Nanomaterials, ed. K. Koumoto and T. Mori, (Springer, Heidelberg, 2013) pp. 255-285.

16. C. Mitterer, J. Solid State Chem. 133, 279 (1997).

17. J. Xu, T. Mori, Y. Bando, D. Golberg, D. Berthebaud, and A. Prytuliak, Mat. Sci. Engineering B 177, 117 (2012).

18. G. Zhao, L. Zhang, L. Hu, H. Yu, G. Min and H. Yu, J. Alloys Compd. 599, 175 (2014).

19. H. Liu, L. Zhang, G. Zhao, G. Feng and G. Min, Ceram. Int. 41, 7745 (2015).

20. T. Miura, K. Yokoyama and M. Takeda, Adv. in Tech. of Mat. and Mat. Proc. J. 5, 70 (2003).

21. H. O. Pierson and A. W. Mullendore, Thin Solid Films 83, 87 (1981). 
22. K. Nakamura, J. Electrochem. Soc. 131(11), 2691 (1984).

23. S. S. Kher and J. T. Spencer, J. Phys. Chem. Solids 59, 1343 (1998).

24. S. S. Kher, J. V. Romero, J. D. Caruso and J. T. Spencer, Appl. Organometal. Chem. 22, 300 (2008).

25. K. Nishiyuki, H. Sawaki, Y. Iwata and M. Naito, Physica C 471, 1189 (2011).

26. H. R. Ott, M. Chernikov, E. Felder, L. Degiorgi, E. G. Moshopoulou, J. L. Sarrao and Z. Fisk, Z. Phys. B 102, 337 (1997). 


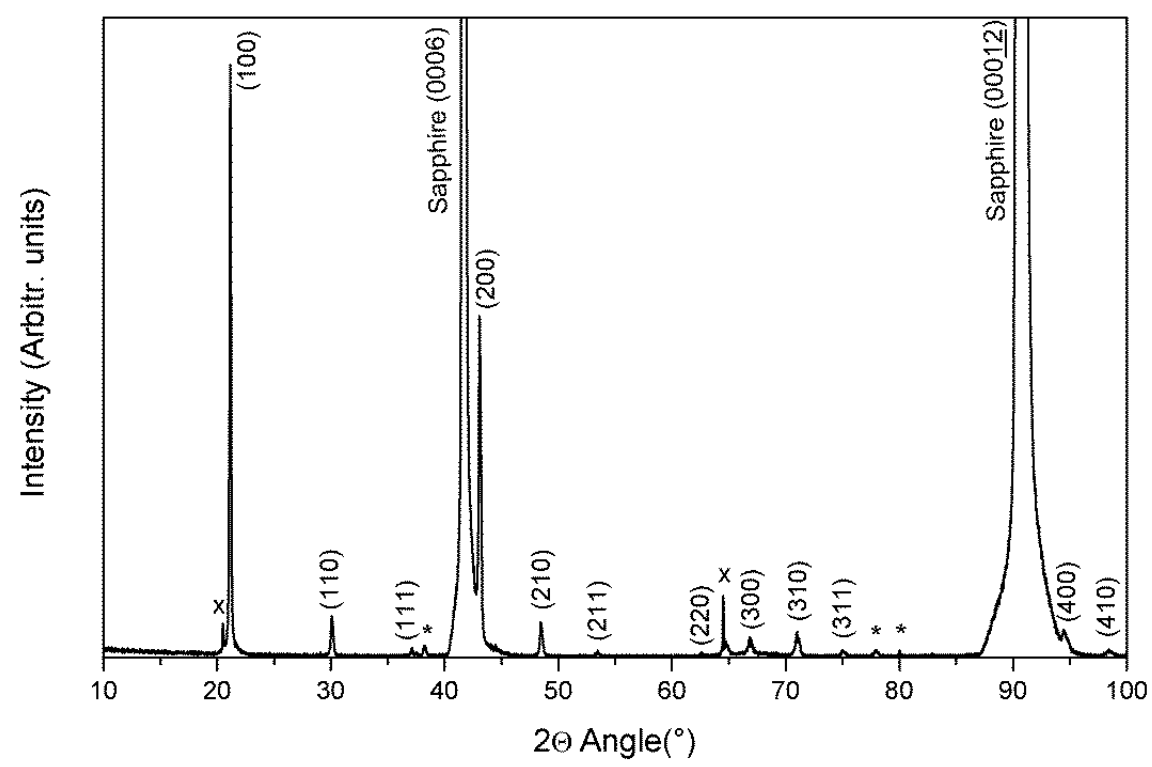

Figure 1. An XRD pattern of a $\mathrm{SrB}_{6}$ film deposited at $950{ }^{\circ} \mathrm{C}$, with the peaks resulting from $\mathrm{SrB}_{6}$ indexed. Peaks marked with $\times$ and $*$ correspond to forbidden peaks of the sapphire substrate and unknown peaks from substrate impurities, respectively. 


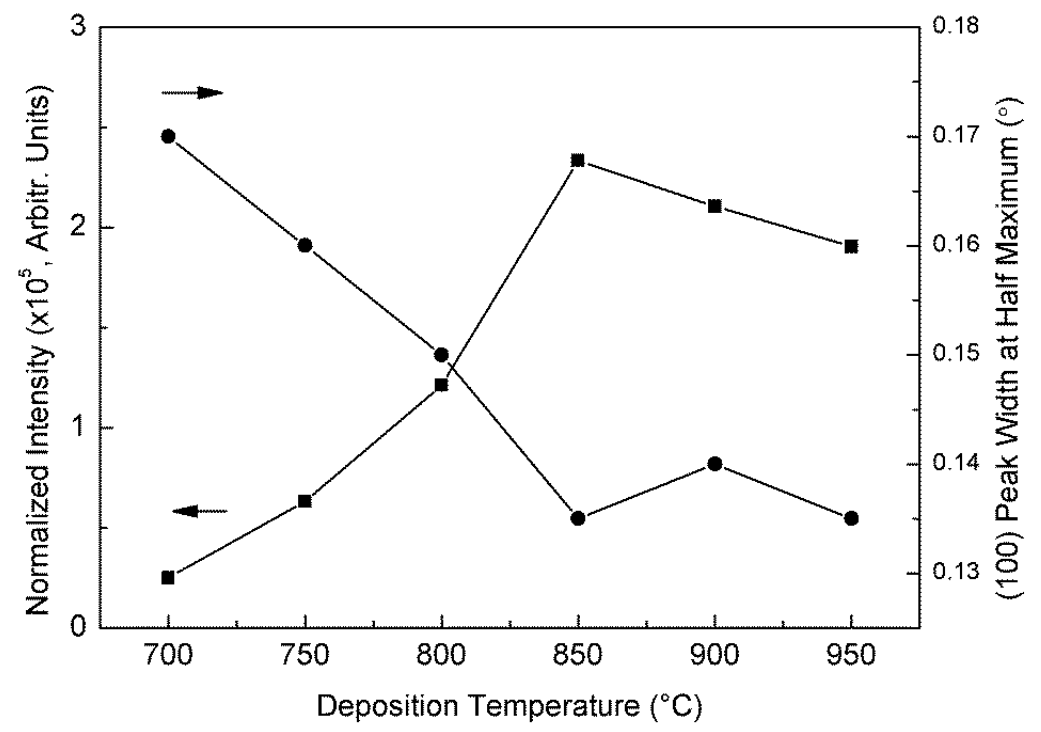

Figure 2. The normalized (100) diffraction peak intensities (squares) and peak widths (circles) of the $\mathrm{SrB}_{6}$ films deposited at different temperatures. 


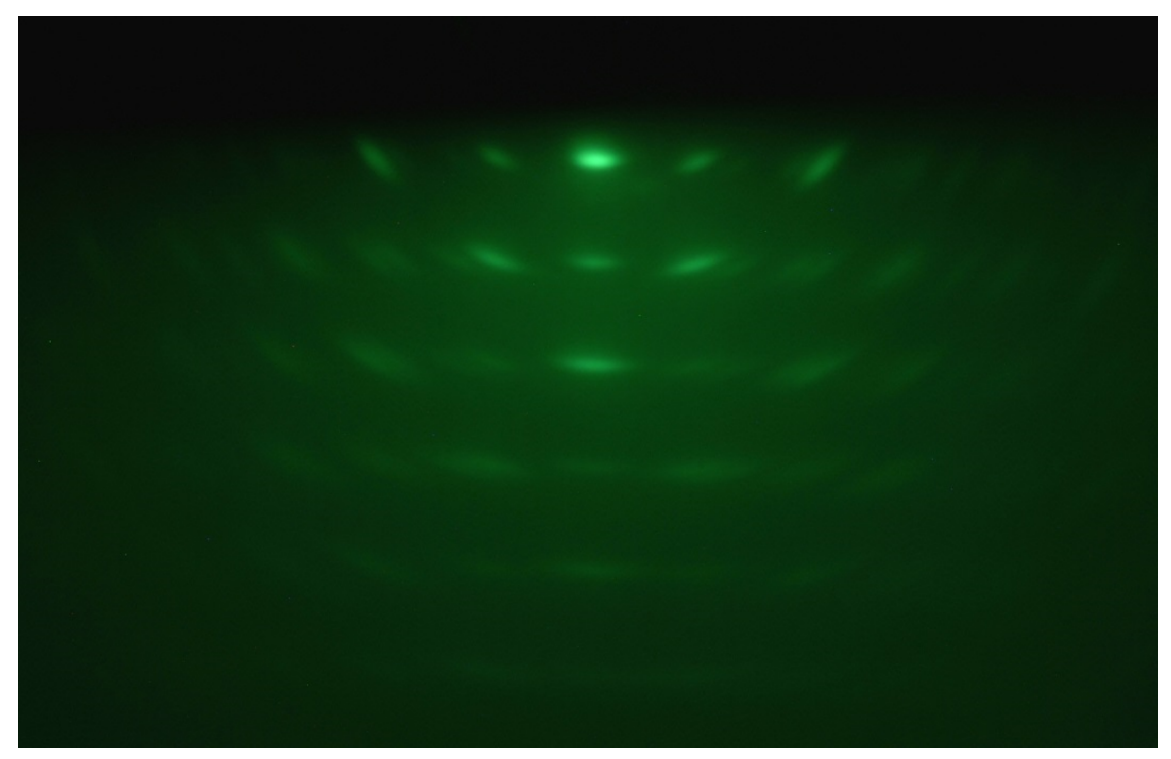

Figure 3. A RHEED pattern obtained from a $\mathrm{SrB}_{6}$ film deposited at $950{ }^{\circ} \mathrm{C}$. 


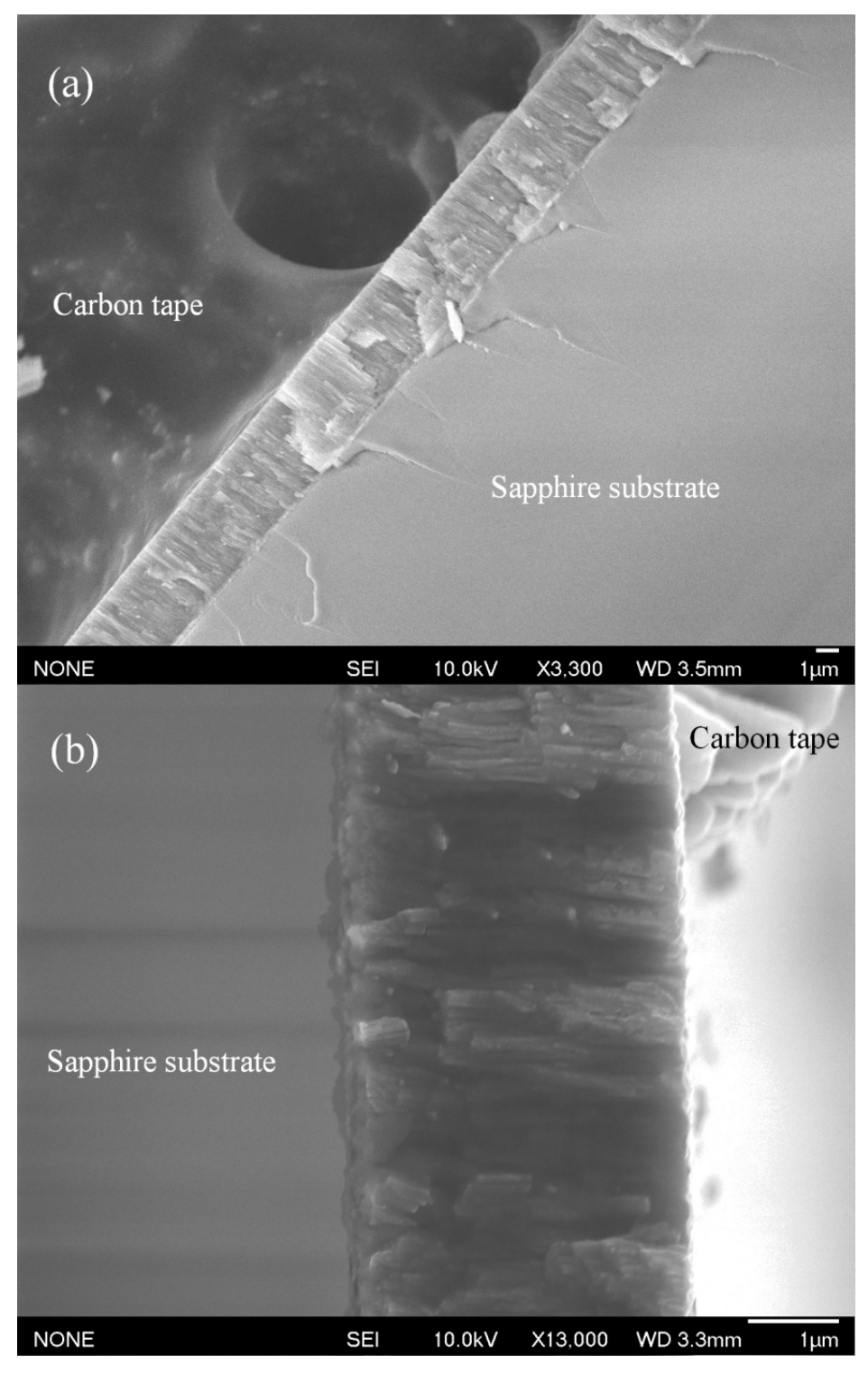

Figure 4. Cross-sectional SEM images of a $\mathrm{SrB}_{6}$ film performed at (a) 3300x and (b) 13000x magnification. 


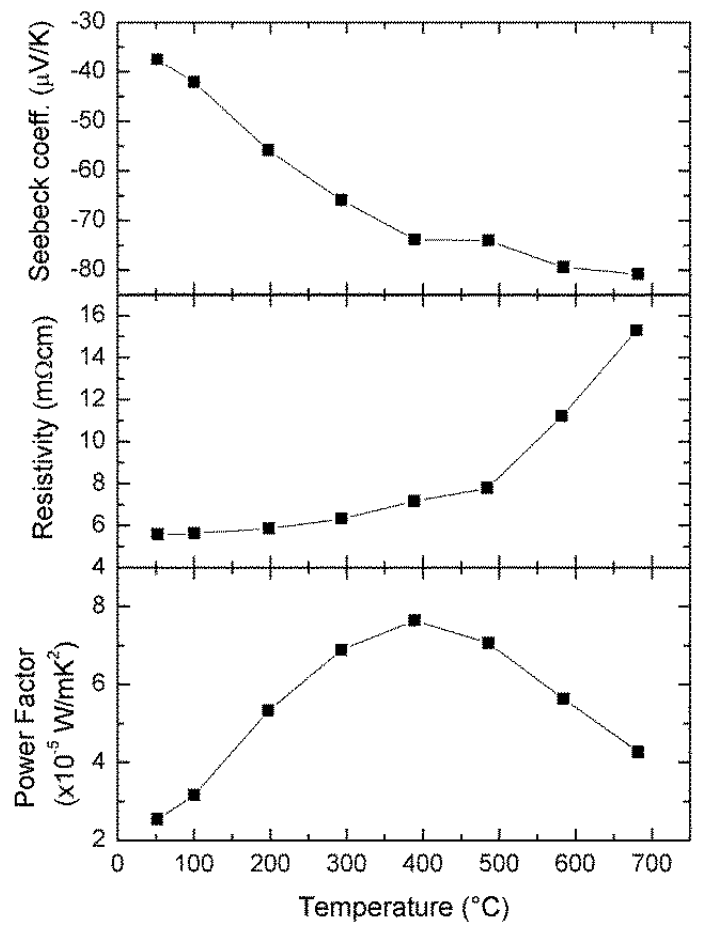

Figure 5. Seebeck coefficient, resistivity and thermoelectric power factor values for the $\mathrm{SrB}_{6}$ films measured between room temperature and $700{ }^{\circ} \mathrm{C}$. 